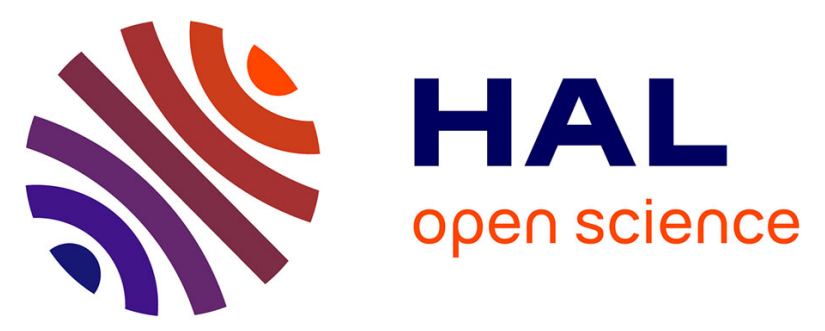

\title{
Développement et validation d'un questionnaire identifiant différentes stratégies comportementales chez les patients lombalgiques chroniques
}

Florian Bailly, Violaine Foltz, Sylvie Rozenberg, Michael Norberg, Joelle Glemarec, Sophie Pouplin, Beatrice Banneville, Christophe Demoulin, Véronique Gaud-Listrat, Bruno Fautrel, et al.

\section{To cite this version:}

Florian Bailly, Violaine Foltz, Sylvie Rozenberg, Michael Norberg, Joelle Glemarec, et al.. Développement et validation d'un questionnaire identifiant différentes stratégies comportementales chez les patients lombalgiques chroniques. Revue du Rhumatisme, 2017, 10.1016/j.rhum.2016.10.003 . hal01480365

\section{HAL Id: hal-01480365 \\ https://hal.sorbonne-universite.fr/hal-01480365}

Submitted on 1 Mar 2017

HAL is a multi-disciplinary open access archive for the deposit and dissemination of scientific research documents, whether they are published or not. The documents may come from teaching and research institutions in France or abroad, or from public or private research centers.
L'archive ouverte pluridisciplinaire HAL, est destinée au dépôt et à la diffusion de documents scientifiques de niveau recherche, publiés ou non, émanant des établissements d'enseignement et de recherche français ou étrangers, des laboratoires publics ou privés. 
Développement et validation d'un questionnaire identifiant différentes stratégies comportementales chez les patients lombalgiques chroniques

Florian Bailly ${ }^{1,2}$; Violaine Foltz ${ }^{1,2}$; Sylvie Rozenberg ${ }^{1,2}$; Michael Norberg ${ }^{3}$; Joelle Glemarec $^{4}$, Sophie Pouplin ${ }^{5}$; Beatrice Banneville ${ }^{1}$, Christophe Demoulin ${ }^{7}$, Véronique Gaud-Listrat $^{8}$, Bruno Fautrel ${ }^{1,2}$; Laure Gossec ${ }^{1,2}$

1 - Service de Rhumatologie, Hôpital Pitié Salpêtrière, AP-HP, 75013 Paris, France ;

2 - Institut Pierre Louis d'Epidémiologie et de Santé Publique, Sorbonne Universités, UPMC Univ Paris 06, Paris 75013, France

3 - Service MPR, Centre hospitalier universitaire vaudois, 1011 Lausanne, Suisse

4 - Centre hospitalier de Nantes, 44093 Nantes, France

5 - Centre hospitalier Charles Nicolle, 76000 Rouen, France

6 - Département des Sciences de la Motricité, Université de Liège, 4000 Liège, Belgique

7 - Service de l'Appareil Locomoteur, CHU de Liège, 4000 Liège, Belgique

8 - AP-HP, Hopital Cochin, Service de Rhumatologie B, 75014 Paris , France

\section{Auteur correspondant :}

Florian Bailly,

Hôpital Pitié-Salpétrière, Service de Rhumatologie, 47-83, boulevard de l'Hôpital 75013 Paris France

florian.bailly@aphp.fr

$+33142177821$ 


\section{Résumé}

Introduction. Identifier différentes stratégies comportementales, adaptées ou inadaptées face à la douleur, permettrait d'optimiser la prise en charge des patients lombalgiques.

Objectif. Elaboration et validation d'un questionnaire identifiant différentes stratégies comportementales chez des patients souffrant de lombalgie chronique nonspécifique.

Méthodes. (1) Constitution d'un questionnaire préliminaire à partir d'une étude qualitative réalisée chez 25 patients lombalgiques (2) Réduction d'items et validation du questionnaire par une étude prospective multicentrique internationale chez des patients lombalgiques chroniques. Une analyse des correspondances multiples a individualisé différents profils comportementaux. Leurs caractéristiques, validité interne et externe ont été évaluées. Suivi longitudinal à 12 mois des thérapeutiques effectuées chez 58 patients. (3) Évaluation de la reproductibilité chez 30 patients.

Résultats: (1) Questionnaire préliminaire comprenant 86 items répartis en 8 domaines de gestion de la douleur. (2) 3 groupes comportementaux ont été identifiés: comportement adapté, détresse émotionnelle, kinésiophobie. Le questionnaire a été réduit à 7 questions (21 items). Le coefficient de Cronbach était modéré (0.47-0.66). La validité externe évaluée par rapport à l'anxiété, la dépression, et les stratégies de coping était bonne. Les patients kinésiophobes avaient plus eu recours au réentraînement à l'effort que ceux avec un comportement adapté, comme attendu (50\% vs $25 \%$, p < 0,05). (3) La reproductibilité était bonne (kappa = 0.70).

Conclusion: Ce nouveau questionnaire simple permet d'identifier 3 groupes comportementaux chez les patients lombalgiques chroniques, afin de guider une prise en charge personnalisée.

Mots-Clés : Lombalgie ; questionnaire ; coping ; comportement ; gestion ; gestion de la douleur; stratégie d'ajustement. 


\section{Introduction}

La lombalgie commune est une pathologie fréquente (prévalence mondiale de 9,7\%), stable entre 1990 et 2010 [1] et est responsable d'un handicap significatif : une évaluation des pathologies responsables des " années de vie avec incapacité » (Years lived with disability) classe les lombalgies comme première cause mondiale de diminution d'années de vie sans incapacité [1].

Pour autant, certains progrès ont déjà été réalisés : le modèle anatomo-clinique initial faisant correspondre une douleur à une lésion rachidienne a été remplacé par une approche « bio-psycho-sociale » par l'Organisation Mondiale de la Santé [2], incluant les causes anatomiques, psychologiques et socioprofessionnelles. Cette approche souligne également la nécessité d'une prise en charge personnalisée et multimodale en raison de l'hétérogénéité des patients, centrée non pas sur la topographie de la douleur mais sur l'ensemble des caractéristiques des patients.

De nombreux traitements ont prouvé leur efficacité dans les lombalgies chroniques: rééducation physique, interventions éducatives, prise en charge multidisciplinaire, thérapies cognitivo-comportementales sont proposées dans les recommandations européennes [3]. Néanmoins choisir le traitement le plus adapté pour chaque patient n'est pas aisé en pratique clinique. Ces recommandations incitent à identifier des sous-groupes de patients pour permettre de mieux cibler les thérapeutiques selon les caractéristiques des patients. Un questionnaire (STarT Back Screening Tool) classant les patients selon le risque de chronicité permet d'améliorer la prise en charge des patients [4] en identifiant les patients à haut risque de chronicité et en les orientant vers une prise en charge selon les résultats obtenus (rééducation, psychologue, prise en charge sociale). Néanmoins le résultat du questionnaire STarT Back Screening Tool est interprété par le médecin, et pas directement par le patient. Aucun conseil d'amélioration de la prise en charge n'est donné au patient, et ceci ne contribue pas à l'éducation thérapeutique du patient lombalgique.

Par ailleurs, l'existence de différentes manières de gérer la douleur et l'importance de la gestion dans l'impact des douleurs ont déjà été soulignées dans différentes études 
$[5,6]$, et ce point doit être pris en compte dans la stratégie thérapeutique des lombalgies [7]. Cette évaluation peut avoir un double but [8]:

- Permettre au médecin d'identifier des modalités de gestion de la douleur inadéquate ou suboptimale, et de ce fait les cibles en termes de prise en charge thérapeutique.

- Permettre au patient d'identifier lui-même d'autres modalités de gestion des activités quotidiennes et de participer activement à la stratégie thérapeutique et à la définition de ses objectifs (objectifs " partagés » en termes d'éducation thérapeutique).

Or aucune méthode de classement, facilement utilisable par les patients et les médecins, centrée sur les comportements face à la gêne fonctionnelle et à la douleur des lombalgiques n'est disponible. Un tel classement permettrait de guider les patients dans la gestion de leur lombalgie, et guider les médecins dans leur prise en charge thérapeutique.

L'objectif de cette étude l'élaboration et la validation d'un questionnaire, autoadministrable, réalisable en pratique courante permettant d'identifier différents profils de gestion de la lombalgie chronique commune chronique . 


\section{Méthodes}

Le développement du présent questionnaire, le BP2 (Back Pain Behavioral Profile), a été réalisé entre 2012 et 2015, sous la coordination d'un groupe de recherche en rhumatologie de l'hôpital de la Pitié Salpêtrière et par la Section Rachis de la Société Française de Rhumatologie. Les règles d'évaluation des critères rapportés par les patients ont été respectées $[9,10]$. Un questionnaire préliminaire a tout d'abord été créé, à partir de verbatims de patients, puis ce questionnaire a été évalué prospectivement et modifié pour obtenir le questionnaire final; enfin la reproductibilité du questionnaire final a été évaluée (figure 1).

\section{1/ Elaboration du questionnaire préliminaire}

À partir des résultats d'une étude qualitative réalisée chez 25 patients [11] (entretiens de groupe semi-structurés réalisés auprès de patients ayant une lombalgie non spécifique chronique), un questionnaire préliminaire a été créé par un groupe de 5 experts rhumatologues. Les différentes modalités de gestion de la douleur avaient été retrouvées dans 8 situations pratiques (Douleur / Activités de la vie quotidienne / Activités physiques et de loisirs / Emotions et moral / Regard des autres / Activités professionnelles / Avenir / Traitements médicamenteux). Ces situations ont constitué les questions. Les propositions de réponses, sous forme de questions à choix multiples, ont été créées à partir des verbatims des patients. Ces items devaient tous respecter le même cadre syntaxique et éviter les négations [10]. Un nombre important d'items, potentiellement redondants, a été sélectionné afin de couvrir l'ensemble des propos retrouvés dans les focus group. L'intégralité des verbatims des focus group a été relue pour vérifier la concordance inverse entre les items créés et les verbatim initiaux. Le questionnaire préliminaire a été évalué par la méthode des entretiens cognitifs [12,13] par 5 patients en face-à-face, et de façon globale par internet pour 8 patients ayant participé aux focus group, et par 3 des experts recrutant les patients. L'évaluation a porté sur la compréhension des questions («le patient comprend-il la question ?»), sur la récupération des informations (« le patient est-il capable de récupérer de sa mémoire les informations requises ? ») et sur le 
jugement («le patient est-il en mesure de porter un jugement?»). Les intitulés des questions pouvaient être reformulés et les items pouvaient être reformulés ou supprimés selon les remarques des participants.

\section{2/ Création du questionnaire définitif}

Une étude prospective multicentrique observationnelle sur 12 mois a été réalisée entre 2012 et 2014 chez des patients ayant consulté en raison de leur lombalgie avec collecte du questionnaire préliminaire et d'autres questionnaires validés, afin de définir et valider les groupes comportementaux, et de sélectionner les items du questionnaire définitif. Cette étude a été approuvée par le comité de protection des personnes (CPP lle de France VI le 24 octobre 2012) fait l'objet d'une déclaration auprès du Comité Consultatif sur le Traitement de l'Information en matière de Recherche dans le domaine de la Santé $\left(n^{\circ} 12.492\right)$ et de la Commission Nationale de l'Informatique et des Libertés CNIL ( $\left.n^{\circ} 1632885\right)$.

\section{Critères d'inclusion}

Les patients inclus avaient: (a) Une lombalgie commune depuis plus de 6 semaines, avec ou sans irradiation, sans indication chirurgicale. (b) La lombalgie était le motif principal de la consultation. (c) Âge supérieur à 18 ans. (d) Consentement écrit après information éclairée. (e) Langue française orale et écrite courante. (f) Absence de chirurgie rachidienne de moins de 3 mois (g) EVA douleur lombaire supérieure à $4 / 10$.

Le recrutement était réalisé de façon non-consécutive dans des centres spécialisés en France, Suisse et Belgique via les membres de la section rachis de la Société Française de Rhumatologie.

\section{Origine et nature des données recueillies}

Les données d'inclusion et le formulaire de consentement écrit étaient recueillis lors d'une consultation médicale. Le cahier patient était complété par les participants par internet via un site sécurisé. Pour les patients n'ayant pas accès à internet, une version papier du questionnaire était envoyée à domicile.

Les données collectées à l'inclusion étaient : 
- Données sociodémographiques (âge, sexe, niveau d'étude, catégorie socioprofessionnelle selon la classification de l'INSEE)

- Caractéristiques des douleurs lombaires (durée, intensité via une Echelle Visuelle Analogique, présence ou non d'une irradiation)

- Questionnaire préliminaire

- Différents questionnaires validés en français: Jugement du patient sur la considération de son état actuel comme satisfaisant: (PASS: Patient Acceptable Symptom State) [14]; Echelle d'Incapacité Fonctionnelle pour l'Evaluation des Lombalgies (EIFEL) [15]; Questionnaire sur les peurs et croyances erronées (Fear Avoidance Belief Questionnaire FABQ) [16] ; Questionnaire de coping (Coping Strategies Questionnaire) [17] ; Questionnaire d'anxiété et de dépression (Hospital Anxiety and Depression Scale HADS) [18]

L'hypothèse était que les groupes constitués avaient des caractéristiques (anxiété, dépression, croyances erronées, coping) différentes, et que les thérapeutiques de prise en charge de ces patients seraient différentes lors du suivi. Les seuils validés des questionnaires HADS ( $\geq 8 / 21$ pour les sous échelle anxiété et dépression) et FABQ ( $\geq 15 / 24$ et $\geq 30 / 42$ pour les sous échelles physique et travail respectivement) ont été utilisés $[19,20]$.

Suivi à 12 mois : Les prises en charge étaient laissées à l'appréciation du clinicien qui n'avait pas connaissance des résultats des questionnaires réalisés. Les patients ayant participé via internet ont été recontactés pour identifier celles réalisées à 12 mois (kinésithérapie, réentrainement à l'effort, utilisation d'antidépresseurs, prise en charge par un psychologue ou un psychiatre, chirurgie du rachis).

3/ Évaluation de la reproductibilité du questionnaire définitif

Le questionnaire définitif a été soumis à 30 patients n'ayant pas participé aux étapes précédentes. Les critères d'inclusion étaient : patients majeurs, parlant et écrivant le français couramment, ayant une lombalgie commune depuis plus de 6 semaines, et n'ayant pas participé à un programme d'éducation thérapeutique ou réentrainement à l'effort. Le questionnaire était complété à deux reprises, espacées de 2 à 7 jours. 
Elaboration du scoring, des conclusions et conseils du questionnaire Le calcul d'un score total devait être défini, en évitant si possible les ex-aequo. Les conclusions et conseils pour chaque groupe ont été rédigés par le groupe d'experts, un infirmier d'éducation thérapeutique et un kinésithérapeute. Leur bonne compréhension a été évaluée par la méthode des entretiens cognitifs $[12,13]$ chez 5 patients.

\section{Méthodologie statistique}

La création des groupes comportementaux a été réalisée par une analyse des correspondances multiples [21] puis une classification hiérarchique [22], à partir de l'ensemble des items du questionnaire préliminaire. La validité des groupes constitués a été évaluée par sa consistance interne (coefficient de Cronbach [23]), validité externe par rapport aux questionnaires de référence collectés, ainsi que sa validité longitudinale. Pour chaque question, l'item le plus discriminant pour chaque groupe était sélectionné par le groupe d'experts, à partir des résultats statistiques. Une question pouvait être supprimée si elle rendait le questionnaire moins discriminant entre les groupes (diminution du coefficient de Cronbach). Le questionnaire définitif devait être de type question à réponse unique (à un profil comportemental correspond un seul item). Le risque alpha de $1^{\text {re }}$ espèce pris en compte était de $5 \%$. La reproductibilité du questionnaire a été évaluée par un Kappa de Cohen [24]. L'analyse statistique a été réalisée à l'aide du logiciel $R$ version 3.0.1.

Rôle de la source de financement :

Cette étude a été financée par une bourse de la Société Française de Rhumatologie (Numéro 2350). 


\section{Résultats}

\section{1/ Elaboration du questionnaire préliminaire}

À partir des quatre focus group réalisés et des 8 domaines de gestion identifiés, 89 items correspondant à des stratégies de gestion de la douleur ont été identifiés. Les items étaient regroupés selon les domaines de gestion pour formuler des questions à choix multiple. La compréhension et la facilité de rappel ont été évaluées, et ont amené à reformuler certains items et en supprimer 2. L'ensemble des 8 questions et 87 items formait le questionnaire préliminaire.

\section{2/ Création du questionnaire définitif}

Cent dix-huit patients ont été inclus, 60 \% étaient des femmes, l'âge moyen était de 50 ans, les patients avaient une lombalgie chronique, en général depuis plusieurs années (tableau I). L'analyse des composantes multiples a identifié le nombre optimal de groupes à 3. Les patients ont été classés dans ces 3 groupes par une classification hiérarchique. La " signification " clinique de ces groupes statistiques a été évaluée par l'analyse des items les plus associés à un groupe (tableau II). Les trois groupes constitués étaient 1/ Patients ayant un comportement adapté en termes de gestion de la douleur: pas de fausses croyances, absence de crainte du mouvement (kinésiophobie), sans affects dépressifs importants. 2/ Patients en détresse émotionnelle : présence de caractéristiques d'anxiété, de dépression et / ou de catastrophisme au premier plan, rendant impossible une perspective d'avenir. 3/ Patients dits kinésiophobes (malgré l'absence d'évaluation formelle de la kinésiophobie) : présence d'une peur du mouvement, de croyances erronées avec diminution importante des mouvements. Le groupe d'experts a ensuite sélectionné l'item le plus adapté pour chaque question et pour chaque groupe afin de constituer le questionnaire définitif. Une des questions (concernant la gestion des traitements médicamenteux) diminuait la consistance interne, elle a été supprimée. Le coefficient de Cronbach pour les groupes comportement adapté, détresse émotionnelle, kinésiophobie était respectivement de 0,$64 ; 0,46$ et 0,57 . 
Compte tenu du faible nombre de patients inclus dans le groupe en détresse émotionnelle, les données de validité externe de ce groupe étaient difficilement interprétables. Les patients avec un comportement adapté avaient significativement plus fréquemment un état acceptable (question PASS), étaient moins anxieux, moins déprimés (score HADS) et avaient des croyances erronées moins fréquentes (score FABQ) par rapport aux patients nécessitant une réassurance physique. De même, la stratégie de gestion positive d'ignorance des douleurs était plus élevée chez les patients avec un comportement adapté tandis que la stratégie de gestion négative (inadaptée) de dramatisation était plus élevée chez les patients kinésiophobes et en détresse émotionnelle selon le score CSQ (tableau III). Lors du suivi longitudinal, 84 patients ont été recontactés, dont 58 ont répondu (59\% des patients dans le groupe comportement adapté, $41 \%$ dans le groupe nécessitant une réassurance physique). Les patients kinésiophobes avaient significativement plus souvent réalisé un réentrainement à l'effort (50\% vs $24 \% \mathrm{p}<0,05)$ que ceux avec un comportement adapté. Les autres thérapeutiques évaluées (kinésithérapie, chirurgie, utilisation d'antidépresseurs, consultation en psychologie ou psychiatrie) n'étaient pas significativement différentes.

\section{3/ Évaluation de la reproductibilité du questionnaire définitif}

Trente patients ont réalisé le test-retest du questionnaire définitif dans un intervalle médian de 5 jours. $70 \%, 13 \%$ et $17 \%$ des patients étaient respectivement dans les groupes comportement adapté, détresse émotionnelle, kinésiophobes. La reproductibilité de la classification dans un groupe comportemental était satisfaisante (kappa de Cohen $=0,70$ ).

La méthode de calcul du résultat devait être simple : la somme des items de chaque groupe comportemental est réalisée, sans pondération des items, le score le plus élevé correspond au profil final. En cas d'égalité (2 profils possibles ?), le patient choisissait la question la plus importante pour lui et l'item sélectionné correspondant au profil comportemental prédominant. Les conclusions et conseils selon les groupes 
ont été créés par le comité de l'étude puis évalués par 5 patients via la méthode des entretiens cognitifs. Plusieurs phrases ont été reformulées. Les patients ont jugé la méthode de notation facile à comprendre et à réaliser. L'ensemble constitue le questionnaire définitif (Figure 2 : questionnaire BP2, et Annexe A, document S1[voir le matériel complémentaire accompagnant la version en ligne de cet article]). 


\section{Discussion}

Cette étude a permis de définir différents groupes comportementaux de gestion de la lombalgie : patients kinésiophobes nécessitant une réassurance physique, patients en détresse émotionnelle nécessitant un soutien psychologique, et enfin patients ayant un comportement adapté. Le groupe majoritaire était celui avec le comportement adapté, tandis que les patients en détresse émotionnelle étaient peu nombreux. Le questionnaire BP2 permet de les identifier facilement et rapidement dans le but d'orienter la prise en charge. De plus nous proposons des conseils de bon sens aux patients afin de rendre le questionnaire plus informatif. La prise en charge conseillée pour les patients avec un comportement adapté serait celle habituellement recommandée de type information, antalgie médicamenteuse et kinésithérapie de rééducation, celle pour les patients en détresse émotionnelle serait conjointement psychologique et physique, et celle des patients kinésiophobes serait une prise en charge physique importante et pluridisciplinaire, par exemple de type réentrainement à l'effort. Ces propositions de prise en charge sont cohérentes avec ce qui a été réalisé chez les patients de cette étude, même si leur validation dans une étude indépendante est nécessaire.

La mise en évidence d'un comportement variable face à la lombalgie, amenant a des conseils et une prise en charge différente, est en accord avec les données de la littérature. Une large étude de cohorte prospective avait identifié le catastrophisme et la kinésiophobie comme prédicteurs indépendants de lombalgie chronique, y compris lorsque ces caractéristiques étaient retrouvées dans une population de sujets n'ayant pas initialement de lombalgie [25]. Ces éléments sont retrouvés ici dans les deux groupes "dysfonctionnels", même si des questionnaires spécifiques de catastrophisme ou de kinésiophobie n'ont pas été réalisés. Lors du suivi à 12 mois en pratique quotidienne, les patients que nous classions comme kinésiophobes avaient plus souvent réalisé un réentrainement à l'effort ce qui conforte la validité des groupes. Cependant, nous n'avons pas vérifié la concordance entre l'opinion du patient et son classement par le questionnaire. L'existence de différentes modalités de gestion de la douleur avait déjà été identifiée également chez des patients ayant 
une lombalgie chronique : Klapow et al. avaient individualisé 3 groupes : « syndrome douloureux », " adaptation positive à la douleur » et " bon contrôle de la douleur » selon leur niveau d'intensité douloureuse, incapacité, dépression, modalités passives de gestion de la douleur (coping) [26,27]. Cette classification, réalisée via une analyse par cluster comme cette étude, retrouvait des caractéristiques proches des groupes ici identifiés, mais n'avait pas permis la création d'un questionnaire utilisable en pratique clinique.

Le faible nombre de patients dans le groupe en détresse émotionnelle correspond à la faible proportion de patients ayant un syndrome dépressif important. Ceci, bien qu'attendu, limite l'interprétation de ce groupe. Néanmoins ces patients doivent être identifiés compte tenu des thérapeutiques spécifiques pour leur prise en charge et pour le risque majeur de chronicisation des douleurs [28] . On notera aussi un nombre différent de patients en détresse émotionnelle dans les différentes phases de notre étude ( $3 \%$ versus $13 \%$ ), indiquant des fluctuations. L'évaluation des trois groupes identifiés et la proportion dans chacun d'entre eux devront être confirmées par une étude dans une population indépendante. Cette évaluation future devrait par ailleurs valider certaines caractéristiques des groupes, comme la kinésiophobie, qui n'avait pas été incluse dans l'analyse initiale.

Récemment, le STarT Back Screening Tool a été développé pour classer les patients en 3 différents groupes, selon le risque (faible, modéré ou élevé) de chronicisation de la douleur. Ce questionnaire, très simple ( 9 items) a été validé et a prouvé son efficacité pour la prise en charge stratifiée de patients ayant une lombalgie en soins primaires [4]. Néanmoins l'identification de patients à risque de passage à la chronicité n'est plus adaptée lorsque les patients avaient une lombalgie chronique. De plus cet outil est destiné aux médecins pour stratifier la prise en charge, mais non directement aux patients afin de les guider.

Les patients inclus dans la constitution et la validation du questionnaire avaient une lombalgie depuis plusieurs années, ce qui correspond à une population particulièrement chronique et ceci peut limiter la validité externe. Néanmoins le large échantillon, le recrutement multicentrique, avec une minorité de patients ayant une prise en charge intensive de type réentrainement à l'effort correspond à une large 
population de patients lombalgiques chroniques recherchant des stratégies thérapeutiques après échec d'une prise en charge initiale. Une étude validant ce questionnaire dans une population de patients lombalgiques subaigus pourrait être intéressante.

L'élaboration et la validation de questionnaires auto-administrés est un domaine de recherche qui a été largement exploré par les rhumatologues en particulier, du fait du caractère subjectif et symptomatique des pathologies rhumatologiques [29-35]. La méthodologie choisie, avec notamment une première partie qualitative et l'utilisation d'avis d'experts peut induire une certaine subjectivité. Dans ce projet, nous avons appliqué des méthodologies reconnues pour élaborer le questionnaire, en se basant sur les patients (patient-derived) et aussi sur l'avis d'experts [29,30,34,35]. Cependant le fait de se baser sur les verbatims de patients pour élaborer le questionnaire comporte une limite liée à l'absence d'éléments difficiles à rapporter comme la recherche de bénéfices secondaires. Nous avons également appliqué des méthodologies reconnues pour la validation du questionnaire $[9,36,37]$. Le choix de la méthodologie statistique (analyse des correspondances multiples) a permis également la constitution de groupes objectifs et validés statistiquement, sans choix subjectifs. Cependant des études ultérieures devront valider les groupes identifiés ici et en particulier le groupe « détresse émotionnelle » dont l'effectif ici était très faible.

Au total, le questionnaire que nous avons développé devrait s'avérer utile à la fois pour les patients et les médecins. Les patients peuvent recevoir un avis sur leur comportement adapté ou inadapté et avoir des conseils globaux pour améliorer ce comportement face à la lombalgie. Les médecins pourraient utiliser ce questionnaire pour adapter la prise en charge thérapeutique, proposer un axe d'éducation thérapeutique ou orienter vers une prise en charge spécifique comme le réentrainement à l'effort. L'utilisation de ce questionnaire paraît particulièrement pertinente pour des médecins non spécialistes du rachis qui peuvent hésiter dans la prise en charge la plus adaptée, ainsi que pour une diffusion large auprès de tous les patients souhaitant améliorer leurs comportements. Ces éléments devront être confirmés dans une étude longitudinale validant l'intérêt de ce questionnaire. 


\section{Déclaration d'intérêts}

Les auteurs déclarent n'avoir aucun lien d'intérêt dans le cadre de cette étude.

\section{Remerciements}

Nous tenons à remercier la Société Française de Rhumatologie qui a subventionné ce travail par une bourse. Nous tenons à remercier la section Rachis reconnue par la Société Française de la Rhumatologie ainsi que les membres qui ont permis le recrutement des patients : Dr Dupeyron, Dr Genevay, Dr Maigne.

\section{Annexe A. Matériel complémentaire}

Du matériel complémentaire (Document S1) accompagnant cet article est disponible sur 


\section{Références}

[1] Vos T, Flaxman AD, Naghavi M. Years lived with disability (YLDs) for 1160 sequelae of 289 diseases and injuries 1990-2010: a systematic analysis for the Global Burden of Disease Study 2010. Lancet 2012;380:2163-96.

[2] World Health Organization. classification internationale des handicaps: déficiences, incapacités et désavantages, un manuel de classification des conséquences des maladies. 1980,204 p.

[3] Airaksinen O, Brox JI, Cedraschi C, et al. Chapter 4 European guidelines for the management of chronic nonspecific low back pain. Eur Spine J 2006;15:192-300.

[4] Hill JC, Whitehurst DG, Lewis M, et al. Comparison of stratified primary care management for low back pain with current best practice (STarT Back): a randomised controlled trial. Lancet 2011;378:1560-71.

[5] Snelgrove S, Liossi C. Living with chronic low back pain: a metasynthesis of qualitative research. Chronic Illn 2013;9:283-301.

[6] Snelgrove S, Edwards S, Liossi C. A longitudinal study of patients' experiences of chronic low back pain using interpretative phenomenological analysis: changes and consistencies. Psychol Health 2013;28:121-38.

[7] Rozenberg S, Foltz V, Fautrel B. Stratégie thérapeutique devant une lombalgie chronique. Rev Rhum 2012;79:A27-31.Treatment strategy for chronic low back pain. Joint Bone Spine. $2012 ; 79: 555-9$

[8] Kirwan J, Heiberg T, Hewlett S, et al. Outcomes from the Patient Perspective Workshop at OMERACT 6. J Rheumatol. 2003;30:868-72.

[9] Boers M, Brooks P, Strand C V, Tugwell P. The OMERACT filter for Outcome Measures 
in Rheumatology. J Rheumatol 1998;25:198-9.

[10] Kirwan JR, Bartlett SJ, Beaton DE, et al. Updating the OMERACT filter: implications for patient-reported outcomes. J Rheumatol 2014;41:1011-5.

[11] Bailly F, Foltz V, Rozenberg S, Fautrel B, Gossec L. The impact of chronic low back pain is partly related to loss of social role: A qualitative study. Joint Bone Spine. $2015 ; 82:: 437-41$.

[12] Drennan J. Cognitive interviewing: verbal data in the design and pretesting of questionnaires. 2003:57-63.

[13] Pincus T, Santos R, Breen A, Burton AK, Underwood M. A review and proposal for a core set of factors for prospective cohorts in low back pain: a consensus statement. Arthritis Rheum 2008;59:14-24.

[14] Pham T, Tubach F. Patient acceptable symptomatic state (PASS). Joint Bone Spine $2009 ; 76: 321-3$.

[15] Coste J, Parc JM Le, Berge E, Delecoeuillerie G, Paolaggi JB. [French validation of a disability rating scale for the evaluation of low back pain (EIFEL questionnaire)]. Rev Rhum Ed Fr 1993;60:335-41.

[16] Chaory K, Fayad F, Rannou F, et al. Validation of the French version of the fear avoidance belief questionnaire. Spine (Phila Pa 1976) 2004;29:908-13.

[17] Irachabal S, Koleck M, Rascle N, Bruchon-Schweitzer M. [Pain coping strategies: French adaptation of the coping strategies questionnaire (CSQ-F)]. Encephale 2008;34:47-53. [18] Lépine JP, Godchau M, Brun P, Lempérière T. [Evaluation of anxiety and depression among patients hospitalized on an internal medicine service]. Ann médico-psychologiques $1985 ; 143: 175-89$.

[19] Burton AK, Waddell G, Tillotson KM, Summerton N. Information and advice to patients 
with back pain can have a positive effect a randomized controlled trial of a novel educational booklet in primary care. 1999;24:2484-91.

[20] Bjelland I, Dahl A a, Haug TT, Neckelmann D. The validity of the Hospital Anxiety and Depression Scale. An updated literature review. J Psychosom Res 2002;52:69-77.

[21] Everitt BS, Howell DC eds. Encyclopedia of Statistics in Behavioral Science. Chichester, UK: John Wiley \& Sons, Ltd; 2005.

[22] Johnson SC. Hierarchical clustering schemes. Psychometrika 1967;32:241-54.

[23] Cronbach LJ. Coefficient alpha and the internal structure of tests. Psychometrika $1951 ; 16: 297-334$.

[24] Cohen J. A coefficient of agreement for nominal scales. Educ Psychol Meas $1960 ; 202: 27-46$.

[25] Picavet HS, Vlaeyen JW, Schouten JS. Pain catastrophizing and kinesiophobia:

Predictors of chronic low back pain. Am J Epidemiol 2002;156:1028-34.

[26] Klapow JC, Slater MA, Patterson TL, Doctor JN, Atkinson JH, Garfin SR. An empirical evaluation of multidimensional clinical outcome in chronic low back pain patients. Pain $1993 ; 55: 107-18$.

[27] Klapow JC, Slater MA, Patterson TL, et al. Psychosocial factors discriminate multidimensional clinical groups of chronic low back pain patients. Pain 1995;62:349-55. [28] Fransen M, Woodward M, Norton R, Coggan C, Dawe M, Sheridan N. Risk factors associated with the transition from acute to chronic occupational back pain. Spine (Phila Pa 1976) $2002 ; 27: 92-8$.

[29] Gossec L, Dougados M, Rincheval N, et al. Elaboration of the preliminary Rheumatoid Arthritis Impact of Disease (RAID) score: a EULAR initiative. Ann Rheum Dis 2009;68:1680-5 
[30] Gossec L, Wit M de, Kiltz U, et al. A patient-derived and patient-reported outcome measure for assessing psoriatic arthritis: elaboration and preliminary validation of the Psoriatic Arthritis Impact of Disease (PsAID) questionnaire, a 13-country EULAR initiative. Ann Rheum Dis 2014;73:1012-9.

[31] Pincus T, Swearingen CJ, Bergman M, Yazici Y. RAPID3 (Routine Assessment of Patient Index Data 3), a rheumatoid arthritis index without formal joint counts for routine care: proposed severity categories compared to Disease Activity Score and Clinical Disease Activity Index Categories. J Rheumatol 2008;35:2136-47.

[32] Fries JF, Spitz PW, Young DY. The dimensions of health outcomes: the health assessment questionnaire, disability and pain scales. J Rheumatol 1982;9:789-93.

[33] Castrejón I, Gossec L, Carmona L. The EULAR Outcome Measures Library: an evolutional database of validated patient-reported instruments. Ann Rheum Dis 2015;74:4756.

[34] Hewlett S, Dures E, Almeida C. Measures of fatigue: Bristol Rheumatoid Arthritis Fatigue Multi-Dimensional Questionnaire (BRAF MDQ), Bristol Rheumatoid Arthritis Fatigue Numerical Rating Scales (BRAF NRS) for severity, effect, and coping, Chalder Fatigue Questionnaire (CFQ), Checklist. Arthritis Care Res (Hoboken) 2011;63 Suppl 1:S263-86.

[35] Sanderson T, Morris M, Calnan M, Richards P, Hewlett S. Patient perspective of measuring treatment efficacy: the rheumatoid arthritis patient priorities for pharmacologic interventions outcomes. Arthritis Care Res (Hoboken) 2010;62:647-56.

[36] Boers M, Kirwan JR, Wells G, et al. Developing core outcome measurement sets for clinical trials: OMERACT filter 2.0. J Clin Epidemiol 2014;67:745-53.

[37] Mokkink LB, Terwee CB, Patrick DL, et al. The COSMIN checklist for assessing the 
methodological quality of studies on measurement properties of health status measurement instruments: an international Delphi study. Qual Life Res 2010;19:539-49. 
Tableau I: Caractéristiques cliniques de la population de l'étude prospective multicentrique de création du questionnaire

\begin{tabular}{|c|c|c|c|}
\hline & $\begin{array}{l}\text { Population étude } \\
\text { prospective } \\
\text { multicentrique ( } \mathrm{n} \\
=118)\end{array}$ & $\begin{array}{l}\text { Sous-groupe } \\
\text { de l'étude } \\
\text { prospective } \\
\text { avec le suivi } \\
\text { longitudinal } \\
(n=58)\end{array}$ & $\begin{array}{l}\text { Population } \\
\text { test-retest } \\
(n=30)\end{array}$ \\
\hline $\begin{array}{l}\text { Age, med }(\min -\max ), \\
\text { années }\end{array}$ & $51(22-77)$ & $49(22-77)$ & $50(23-78)$ \\
\hline Femmes, N (\%) & $71(60)$ & $31(53)$ & $18(60)$ \\
\hline $\begin{array}{l}\text { Durée des douleurs } \\
\text { lombaires med (min }- \text { max), } \\
\text { années }\end{array}$ & $8,0(0,3-56)$ & $8,0(0,5-40)$ & $2,0(0,2-20)$ \\
\hline $\begin{array}{l}\text { Présence d'une irradiation } \\
\text { dans un membre inférieur, } \mathrm{N} \\
(\%)\end{array}$ & $88(75)$ & $45(77)$ & $17(57)$ \\
\hline $\begin{array}{l}\text { EVA douleur lombaire } / 10, \\
\operatorname{med}(\min -\max )\end{array}$ & $6,0(4-10)$ & $4,8(1-9)$ & $5,6(3-9)$ \\
\hline $\begin{array}{l}\text { Participation à } \quad \text { un } \\
\text { réentrainement à l'effort, } \\
\text { école du dos, } \mathrm{N}(\%)\end{array}$ & $44(37)$ & $26(45)$ & $0(0)$ \\
\hline Arrêt de travail, N (\%) & $32 / 105(30)$ & $12 / 51(24)$ & $7 / 26(27)$ \\
\hline
\end{tabular}


Tableau II : items les plus fortement associés aux groupes comportementaux

\begin{tabular}{|c|c|c|}
\hline & $\begin{array}{l}\text { \% des patients } \\
\text { du groupe } \\
\text { sélectionnant } \\
\text { cet item }\end{array}$ & $\begin{array}{l}\% \text { des patients } \\
\text { des autres } \\
\text { groupes } \\
\text { sélectionnant } \\
\text { cet item }\end{array}$ \\
\hline \multicolumn{3}{|c|}{$\begin{array}{l}\text { Groupe 1: Patients avec un comportement adapté (60\% de la population } \\
\text { initiale) }\end{array}$} \\
\hline $\begin{array}{l}\text { J'ai appris à faire avec mon mal de dos dans mes } \\
\text { activités de la vie quotidienne }\end{array}$ & $67 \%$ & $32 \%$ \\
\hline $\begin{array}{l}\text { J'accepte l'avenir, même si je sais que je pourrais } \\
\text { avoir encore mal }\end{array}$ & $70 \%$ & $25 \%$ \\
\hline \multicolumn{3}{|c|}{ Groupe 2 : Patients en détresse émotionnelle (3\% de la population initiale) } \\
\hline $\begin{array}{l}\text { J'ai l'impression d'être un poids pour mon } \\
\text { entourage }\end{array}$ & $100 \%$ & $13 \%$ \\
\hline Je suis déprimé par mon état douloureux & $100 \%$ & $16 \%$ \\
\hline \multicolumn{3}{|c|}{ Groupe 3 : Patients kinésiophobes (37 \% de la population initiale) } \\
\hline Mon avenir me fait peur & $59 \%$ & $10 \%$ \\
\hline $\begin{array}{l}\text { Je suis bloqué dans les gestes de la vie } \\
\text { quotidienne par mon mal de dos }\end{array}$ & $50 \%$ & $17 \%$ \\
\hline
\end{tabular}


Tableau III : Validité externe des 3 groupes comportementaux du questionnaire.

\begin{tabular}{|c|c|c|c|c|}
\hline Variables & $\begin{array}{l}\text { Comportemen } \\
\mathrm{t} \text { adapté (n } \\
=71)\end{array}$ & $\begin{array}{l}\text { Détresse } \\
\text { émotionnelle } \\
(n=3)\end{array}$ & $\begin{array}{l}\text { Kinésiophobe } \\
\mathrm{s}(\mathrm{N}=44)\end{array}$ & $\mathrm{p}$ \\
\hline EVA douleur initiale /10 & $6.2(1.4)$ & $4.3(0.6)$ & $6.3(1.6)$ & 0.092 \\
\hline Arrêt de travail & $27 \%(45)$ & $33 \%(58)$ & $34 \%(48)$ & 0.79 \\
\hline PASS & $35 \%(48)$ & $33 \%(58)$ & $7 \%(25)^{*}$ & $0.003^{\mathfrak{E}}$ \\
\hline $\begin{array}{l}\text { HADS Seuil d'anxiété } \geq \\
8 / 21\end{array}$ & $42 \%(50)$ & $\begin{array}{l}100 \% \\
(0)\end{array}$ & $91 \%(29)$ & $\begin{array}{l}<0.001 \\
\varepsilon\end{array}$ \\
\hline $\begin{array}{l}\text { HADS Seuil de dépression } \\
\geq 8 / 21\end{array}$ & $15 \%(36)$ & $100 \%(0)$ & $65 \%(48)$ & $\begin{array}{l}<0.001 \\
£\end{array}$ \\
\hline FABQ Physique $\geq 15 / 24$ & $41 \%(50)$ & $33 \%(58)$ & $65 \%(48)^{* \star}$ & $0.037^{\mathfrak{E}}$ \\
\hline FABQ Travail $\geq 30 / 42$ & $18 \%(39)$ & $0 \%(0)$ & $38 \%(49)^{\star *}$ & $0.048^{\varepsilon}$ \\
\hline $\begin{array}{lll}\text { Incapacité } & \text { EIFEL } & \text { à } \\
\text { l'inclusion / } 24 & & \end{array}$ & $6.9(3.7)$ & $12.7(2.3)$ & $12.3(3.4)$ & 0.304 \\
\hline CSQ Positif Ignorance /16 & (3.5) & $14.3(3.1)$ & $9.1(2.2)^{* * *}$ & $0.001^{\varepsilon}$ \\
\hline $\begin{array}{l}\text { CSQ Négatif Distraction / } \\
20\end{array}$ & $10.7(3.7)$ & $12.0(3.6)$ & $10.6(3.2)$ & 0.761 \\
\hline CSQ Négatif Dramatisation & $8.1(2.6)$ & $10.3(1.2)$ & $11.4(2.5)^{\star * \star}$ & $\begin{array}{l}<0.001 \\
\varepsilon\end{array}$ \\
\hline $\begin{array}{l}\text { CSQ Positif } \\
\text { Réinterprétation /16 }\end{array}$ & $6.3(2.6)$ & $4.7(1.2)$ & $5.5(1.8)$ & 0.344 \\
\hline CSQ Négatif Prière / 12 & $5.0(2.7)$ & $6.3(4.2)$ & $5.9(2.6)$ & 0.080 \\
\hline
\end{tabular}

Légende : $E V A=$ Echelle visuelle analogique. $H A D S=$ Hospital anxiety and depression scale. $F A B Q=$ Fear avoidance and beliefs questionnaire. EIFEL = échelle d'incapacité fonctionnelle pour l'évaluation des lombalgies. CSQ = Coping strategies questionnaire. Les résultats sont présentés en moyenne ou pourcentage (écart type). p significatif (test de kruskal wallis) : ${ }^{*} p<0,01 ;{ }^{* *} p<0,05 ;{ }^{* * *} p<0,001$. 
Figure 1 : Schéma de l'étude

Figure 2: Questionnaire sur la gestion de mes douleurs lombaires 


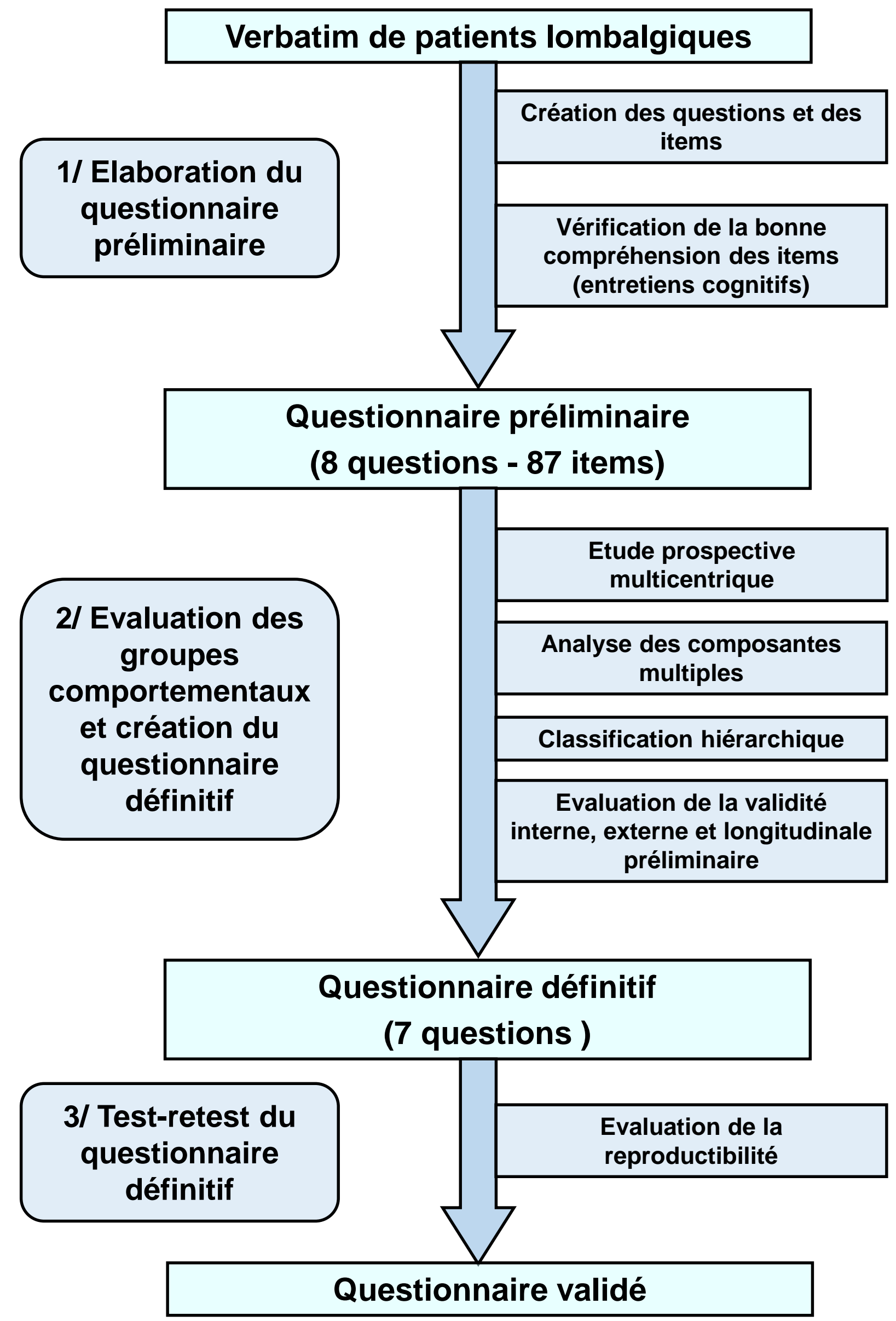


Ce questionnaire permet de mieux comprendre la façon dont vous gérez vos douleurs lombaires. Pour chaque question, sélectionnez la phrase qui vous correspond le mieux en ce moment.

\section{Question 1 - Mes douleurs du dos}

J'ai l'impression de plonger à cause de la douleur.

$\triangle$ Je fais avec mes douleurs.

O Je crains que mes douleurs ne s'arrêtent jamais.

\section{Question 2 - Mes activités de la vie quotidienne}

$\triangle$ J'ai appris à faire avec mon mal de dos dans mes activités de la vie quotidienne.

$\square$ Je ne fais plus aucune activité de la vie quotidienne.

O J'ai dû arrêter certaines activités de la vie quotidienne.

\section{Question 3 - Mes émotions et mon moral}

$\square$ Moralement, j'ai l'impression d'être enfermé dans un cercle vicieux.

O Moralement je $n$ 'arrive pas à faire face.

$\triangle$ Moralement avec ma douleur ce n'est pas toujours facile, mais je fais avec.

\section{Question 4 - Mes activités physiques de loisirs et de sports}

$\square$ Je ne fais plus rien à cause de mon dos.

$\triangle$ J'ai appris à faire des activités physiques avec mon mal de dos.

O J'ai peur de bouger au cours de mes activités physiques.

\section{Question 5 - A propos de l'avenir et de mon mal de dos}

$\triangle$ J'accepte l'avenir, même si je sais que je pourrai avoir encore mal.

O J'ai des craintes pour l'avenir.

J'ai du mal à me projeter dans le futur.

\section{Question 6 - Le regard des autres, par rapport à mon mal de dos}

$\square$ J'ai l'impression d'être un poids pour mon entourage.

$\triangle$ Je me sens soutenu par mon entourage.

O J'ai le sentiment que mon entourage minimise mes douleurs.

\section{Question 7 - Mes activités professionnelles}

$\triangle$ J'ai appris à faire mon travail avec mon mal de dos.

$\square$ Je ne travaille plus à cause de mon dos.

O Je travaille mais j'ai peur que le travail augmente ma douleur.

Nombre de $\triangle$ :

Nombre de $\square$ :

Nombre de $\mathrm{O}$ :

Si vous êtes ex-aequo pour 2 symboles, déterminez la question la plus importante pour vous :

Question numéro: 